\title{
Empirical Value at Risk for Weak Dependent Random Variables
}

\author{
Ali Kabui ${ }^{1} \&$ Samir Ben Hariz ${ }^{1}$ \\ ${ }^{1}$ Laboratoire de Statistique et Processus, Université du Maine, France \\ Correspondence: Ali Kabui, Laboratoire de Statistique et Processus, Université du Maine, 72 Av. Olivier Messiaen, \\ 72085 Le Mans Cedex 9, France. E-mail: ali.kabui.etu@univ-lemans.fr
}

Received: October 7, 2012 Accepted: November 12, 2012 Online Published: December 19, 2012

doi:10.5539/ijsp.v2n1p24 URL: http://dx.doi.org/10.5539/ijsp.v2n1p24

\begin{abstract}
In this work, we study the empirical estimator of the Value at Risk (VaR for short) for weak dependent observations. Our approach uses the oscillation of the empirical process under hypothesis of moment's inequality. We provide general conditions which ensure the convergence of the empirical estimator of the VaR. We also prove a central limit theorem (CLT) for the difference. We perform some simulations for different sequences to illustrate our results. Finally, we apply the results for different sequences under assumptions of mixing or covariance.
\end{abstract}

Keywords: Value at Risk $(V a R)$, modulus of continuity, empirical process, quantile function, moment's inequality, dependent random variables

\section{Introduction}

The Value at Risk $V a R$ is a method to evaluate financial risks. It summarizes the risks of loss in a unique number and aggregating the risks of market through several classes of financial assets (stocks, bonds, etc.).

The $V a R$ is a probabilistic measure of the possible loss for a given horizon. It represents a level of loss, for a financial position or a portfolio, which will be exceeded during a given period only with a chosen typically small probability.

The $\operatorname{VaR}$ is obviously neither the loss which one can expect nor the maximum loss which one may suffer, but a level of loss which will be exceeded only with a level of a fixed probability $q$.

Definition 1 ( $P \& L$ and loss function) Let $P_{t}$ be the value of a portfolio of assets at time $t$. Then the variation of the value of this portfolio over the interval $[t, t+T]$, is called the profit-and-loss $(\mathrm{P} \& \mathrm{~L})$ function:

$$
\triangle P_{t} \equiv P_{t+T}-P_{t}
$$

and the function

$$
X_{t}: \equiv-\triangle P_{t}
$$

is called the loss function.

In practice, we decide to fix $T$ (e.g. one day or one week), yet $\Delta P_{t} \equiv P_{t+1}-P_{t}$.

Definition 2 (Value at Risk) The Value at Risk $\operatorname{VaR}(q)$ of a portfolio of assets for a period $[t, t+1]$ at the confidence level $q \in(0,1)$ is given by the smallest number $x$ such that the probability that the loss $X_{t}$ exceeds $x$ is no larger than $(1-q)$. Formally

$$
\operatorname{VaR}(q) \equiv \inf \left\{x: \mathbb{P}\left(X_{t}>x\right) \leq 1-q\right\}
$$

or

$$
\operatorname{VaR}(q) \equiv F_{t}^{-1}(q)=\inf \{x: F(x) \geq q\}:=\xi .
$$

where $F_{t}(x)=\mathbb{P}\left(X_{t} \leq x\right), x \in \mathbb{R}$ is the distribution function of $X_{t}$ and $F_{t}^{-1}$ its quantile function.

Definition (1.1) clearly shows that the knowledge of the distribution function (in short $d f$ ) of the r.v $X$ can determine the $\operatorname{VaR}(q)$. Often the function $F$ is assumed to be normal. However a lot of financial practitioners use historical distributions which are far from being normally distributed (see e.g. Cont, 2001). Moreover, in general, the historical data have an intertemporally dependent structures. Indeed the assumption that the variables $\left(X_{i}\right)_{1 \leq i \leq n}$ (denote the variations $\left(-\triangle P_{i}\right)_{1 \leq i \leq n}$ in the value of a portfolio over the $n$ periods) are i.i.d, is not easily satisfied 
in practice. Hence the feeling of the need of taking into account a possible dependence structure or an effect of memory in the observations. In order to model and measure this memory aspect in the data, we consider two cases: correlations or mixing coefficients.

So the main objective of this paper is to provide ways which allow to tackle the issue of estimation of the $V a R$ in the cases where there is either a lack of parameterizations of $F$ or some weak dependency among the data. To do so, we use the empirical distribution function $F_{n}(x)=\frac{1}{n} \sum_{i=1}^{n} \mathbb{I}_{\left(X_{i} \leq x\right)}$, where $x \in \mathbb{R}$ and $\mathbb{I}$ is the indicator function, for a stationary sequence of dependent real-valued random variables $\left(X_{i}\right)_{1 \leq i \leq n}$ to estimate the $\operatorname{VaR}$.

The empirical estimator of the $\operatorname{VaR}(\widehat{\operatorname{VaR}})$ (see e.g. Dowd, 2001) is defined by:

$$
\widehat{\operatorname{VaR}}(q) \equiv F_{n}^{-1}(q) \equiv \inf \left\{x: F_{n}(x) \geq q\right\} .
$$

We note that if we order the independent random variables $X_{n, 1} \leq X_{n, 2} \leq \ldots \leq X_{n, n}$ then $\widehat{\operatorname{VaR}}_{e}(q)$ can be written as

$$
\widehat{\operatorname{VaR}}(q)=X_{n, s}, \quad s=[n q]+1 .
$$

where $[a]$ is the integer part of $a$.

Next let us recall the definitions of some mixing coefficients which are criteria needed to introduce dependency measures between variables.

Let $(\Omega, \mathcal{K}, P)$ be a probability space and let $\mathcal{A}, \mathcal{B}$ be two sub $\sigma$-algebras of $\mathcal{K}$. We define:

1) The $\alpha$-mixing coefficient by:

$$
\alpha(\mathcal{A}, \mathcal{B})=\sup _{A \in \mathcal{A}, B \in \mathcal{B}}|P(A \cap B)-P(A) P(B)| .
$$

2) The $\rho$-mixing coefficient by:

$$
\rho(\mathcal{A}, \mathcal{B})=\sup _{f \in L_{2}(\mathcal{A}), g \in L_{2}(\mathcal{B})}|\operatorname{corr}(f, g)|,
$$

where $\operatorname{corr}(f, g)=\frac{\operatorname{Cov}(f, g)}{\sqrt{\operatorname{Var}(f)} \sqrt{\operatorname{Var}(g)}}$.

3) The $\varphi$-mixing coefficient by:

$$
\varphi(\mathcal{A}, \mathcal{B})=\sup _{A \in \mathcal{A}, B \in \mathcal{B}}\left|\frac{P(A \cap B)}{P(A)}-P(B)\right| .
$$

Finally, we say that a stationary sequence $\left(X_{i}\right)_{i \in \mathbb{Z}}$ is strong mixing or $\alpha$-mixing, if

$$
\alpha_{n}=\alpha\left(\sigma\left(X_{i}, i \leq 0\right), \sigma\left(X_{i}, i \geq n\right)\right) \rightarrow_{n \rightarrow \infty} 0 .
$$

The paper is organized as follows. The section 2 is related to the notion of oscillation of an empirical process which is defined for each $f_{x} \in \mathcal{F}$ by:

$$
Z_{n}\left(f_{x}\right)=\frac{1}{\sqrt{n}} \sum_{i=1}^{n}\left[f_{x}\left(X_{i}\right)-\mathbb{E}\left(f_{x}\left(X_{i}\right)\right)\right]=\sqrt{n}\left[F_{n}(x)-F(x)\right]
$$

where $\mathcal{F}$ is the set of characteristic functions of intervals of the form $(-\infty, x)$ for any $x \in \mathbb{R}$. We study the mean of the modulus of continuity of the empirical process defined by

$$
W(n, \delta):=\mathbb{E}\left(\sup _{\left\|f_{x}-f_{y}\right\|_{v} \leq \delta}\left|Z_{n}\left(f_{x}-f_{y}\right)\right|\right)
$$

where $\left\|f_{x}\right\|_{v}=\left(\mathbb{E}\left|f_{x}\right|^{v}\right)^{\frac{1}{v}}$. Our method is inspired by the work by Ben Hariz (2005) who studied the stochastic equicontinuity of empirical processes indexed by a family of functions. 
In the section 3, which is the main part of this work, we prove the consistency as well as a central limit theorem for the $\widehat{V a R}$, i.e.

$$
\sqrt{n}\left(\xi_{n}-\xi\right) \longrightarrow^{d} \mathcal{N}\left(0, \frac{\sigma_{\infty}^{2}(\xi)}{f^{2}(\xi)}\right)
$$

where

$$
\sigma_{\infty}^{2}(\xi)=\sum_{i \in \mathbb{Z}} \operatorname{Cov}\left(\mathbb{I}_{\left(X_{1} \leq \xi\right)}, \mathbb{I}_{\left(X_{i+1} \leq \xi\right)}\right)=\operatorname{Var}\left(\mathbb{I}_{\left(X_{1} \leq \xi\right)}\right)+2 \sum_{i=1}^{+\infty} \operatorname{Cov}\left(\mathbb{I}_{\left(X_{1} \leq \xi\right)}, \mathbb{I}_{\left(X_{i+1} \leq \xi\right)}\right)
$$

is assumed to satisfy $0<\sigma_{\infty}^{2}(\xi)<\infty$.

In the section 4, several applications are discussed. Finally, the section 5 is devoted to simulations which illustrate the results.

\section{Oscillation of the Empirical Process}

First let us introduce the following assumptions: $F$.

$H(X):\left(X_{i}\right)_{1 \leq i \leq n}$ is a stationary sequence of real-valued random variables with a common distribution function

$H(p, X)$ : For all positive real numbers $2 \leq v<p<r \leq \infty$ and for any $\varepsilon>0$, there exists a positive constant $D=D(\varepsilon, p, v, r)<\infty$ such that for any $f \in \mathcal{F}$

$$
\mathbb{E}\left|Z_{n}(f)\right|^{p} \leq D\left(\|f\|_{v}^{p}+n^{1+\varepsilon-\frac{p}{2}}\|f\|_{r}^{p}\right) .
$$

$H(F): F$ is continuous in $\mathcal{I}=\left[\xi-a_{n}, \xi+a_{n}\right]$ where $0<a_{n} \rightarrow_{n \rightarrow \infty} 0$, and $F$ has a density function $f$ which is continuous and $0<f(\xi)<\infty$.

For $0<b_{n} \rightarrow_{n \rightarrow \infty} 0$ we denote,

$$
a_{n} \ll b_{n} \Leftrightarrow\left\{a_{n}<b_{n} \text { and } \frac{a_{n}}{b_{n}} \rightarrow_{n \rightarrow \infty} 0\right\} .
$$

In the proofs $C$ denote constant where values may change from one line to another. We will now focus on the modulus of continuity of an empirical process $\left(X_{i}\right)_{1 \leq i \leq n}$.

Theorem 1 Under conditions $H(X)$ and $H(p, X)$, there exists $C=C(\varepsilon, p, v, r)<\infty$ such that for $\delta>n^{\frac{\frac{1+\varepsilon}{p}-1}{\left(1+\frac{1}{p}-\frac{1}{r}\right)}}$,

$$
W(n, \delta) \leq C \cdot\left(n^{-\frac{1}{2}+\frac{2+\varepsilon-\frac{p}{r}}{p+1-\frac{p}{r}}}+\delta^{\left(1-\frac{v}{p}\right)}\right) .
$$

If in addition $\varepsilon<\frac{p}{2}\left(1-\frac{1}{p}+\frac{1}{r}\right)-1$ and $\delta=\delta_{n} \rightarrow 0$, then

$$
\lim _{n \rightarrow \infty} W\left(n, \delta_{n}\right)=0 .
$$

\section{Remark}

- When $r=p$ the result becomes for $\delta>n^{\frac{1+\varepsilon-p}{v p}}$,

$$
W(n, \delta) \leq C \cdot\left(\ln n \cdot n^{-\frac{1}{2}+\frac{1+\varepsilon}{p}}+\delta^{\left(1-\frac{v}{p}\right)}\right) .
$$

- If $F$ is $L$-Lipschitz, then for $\delta_{0}>\frac{1}{C(v, L)} n^{\frac{\frac{1+\varepsilon}{p}-1}{1+\frac{1}{p}-\frac{1}{r}}}$,

$$
\mathbb{E}\left[\sup _{|x-y| \leq \delta_{0}}\left|Z_{n}\left(f_{x}-f_{y}\right)\right|\right] \leq C \cdot\left(n^{-\frac{1}{2}+\frac{2+\varepsilon-\frac{p}{r}}{p+1-\frac{p}{r}}}+C(v, p, L) \cdot \delta_{0}^{\left(\frac{1}{\nu}-\frac{1}{p}\right)}\right) .
$$

Proof of Theorem 1. Let $N(k)=N_{\Gamma .\rceil}\left(2^{-k},\|.\|_{v}, \mathcal{F}\right), k \in \mathbb{N}$ (the bracketing number) be the minimal number of brackets which are of a norm $\|.\|_{v}$ less than or equal $2^{-k}$ needed to cover $\mathcal{F}$. As $N(k) \leq 2.2^{v k}$ is finite (see e.g. Van der Vaart \& Wellner, 1996, ex 2.5.4 in p. 129), there exists a finite sequence

$$
\left\{f_{x_{k}(i)}, \Delta_{x_{k}(i)}=\mathbb{I}_{\left(x_{k}(i) \leq . \leq x_{k}(i+1)\right)}\right\}_{1 \leq i \leq 2^{v k}}
$$


such that:

1) $\left\|\Delta_{x_{k}(i)}\right\|_{\nu} \leq 2^{-k}$,

2) $\forall f_{x} \in \mathcal{F}, \exists i:\left|f_{x}-f_{x_{k}(i)}\right| \leq \Delta_{x_{k}(i)}$.

We set $\left(\pi_{k}(f), \Delta_{k}(f)\right)$ the first pair $\left(f_{x_{k}(i)}, \Delta_{x_{k}(i)}\right)$ which satisfies $\left|f_{x}-f_{x_{k}(i)}\right| \leq \Delta_{x_{k}(i)}$. Let $q_{0}, k$ and $q_{1} \in \mathbb{N}$ such that $q_{0} \leq k \leq q_{1}$, we define for $1 \leq i \leq 2^{v q_{0}}$,

$$
E_{i}=\left\{f \in \mathcal{F}: \pi_{q_{0}}(f)=f_{x_{q_{0}}(i)}\right\},
$$

then the sets $E_{i}$ form a partition of $\mathcal{F}$. For $\delta \sim 2^{-q_{0}} \Leftrightarrow q_{0} \sim-\frac{\ln \delta}{\ln 2}$, we define:

$$
F_{i, j}=\left\{\left(f_{x}, f_{y}\right) \in \mathcal{F} \times \mathcal{F}: f_{x} \in E_{i}, f_{y} \in E_{j},\left\|f_{x}-f_{y}\right\|_{v} \leq \delta\right\} .
$$

Let now $\Lambda=\left\{(i, j): F_{i, j} \neq \emptyset\right\}$. For every pair $(i, j) \in \Lambda$, we fix an element of $F_{i, j}$ and denote this pair $\left(\phi_{i, j}, \psi_{i, j}\right)$. Let $\left(f_{x}, f_{y}\right)$ be a pair satisfying $\left\|f_{x}-f_{y}\right\|_{v} \leq \delta$, then $\left(f_{x}, f_{y}\right) \in F_{i, j}$ for some $(i, j) \in \Lambda$. We write

$$
f_{x}-f_{y}=f_{x}-\pi_{q_{0}}\left(f_{x}\right)+\pi_{q_{0}}\left(f_{x}\right)-\phi_{i, j}+\phi_{i, j}-\psi_{i, j}+\psi_{i, j}-\pi_{q_{0}}\left(f_{y}\right)+\pi_{q_{0}}\left(f_{y}\right)-f_{y}
$$

but $\pi_{q_{0}}\left(f_{x}\right)=\pi_{q_{0}}\left(\phi_{i, j}\right)$ and $\pi_{q_{0}}\left(f_{y}\right)=\pi_{q_{0}}\left(\psi_{i, j}\right)$ since $f_{x}, \phi_{i, j} \in E_{i}, f_{y}, \psi_{i, j} \in E_{j}$. Consequently:

$$
\sup _{\left\|f_{x}-f_{y}\right\|_{v} \leq \delta}\left|Z_{n}\left(f_{x}-f_{y}\right)\right| \leq 4 \sup _{f_{x} \in \mathcal{F}}\left|Z_{n}\left(f_{x}-\pi_{q_{0}}\left(f_{x}\right)\right)\right|+\sup _{(i, j) \in \Lambda}\left|Z_{n}\left(\phi_{i, j}-\psi_{i, j}\right)\right|
$$

That gives by applying the expectation:

$$
\begin{aligned}
\mathbb{E}\left(\sup _{\left\|f_{x}-f_{y}\right\|_{\nu} \leq \delta}\left|Z_{n}\left(f_{x}-f_{y}\right)\right|\right) & \leq 4 \mathbb{E}\left(\sup _{f_{x} \in \mathcal{F}}\left|Z_{n}\left(f_{x}-\pi_{q_{0}}\left(f_{x}\right)\right)\right|\right)+\mathbb{E}\left(\sup _{(i, j) \in \Lambda}\left|Z_{n}\left(\phi_{i, j}-\psi_{i, j}\right)\right|\right) \\
& \equiv 4 E_{1}+E_{2} .
\end{aligned}
$$

In order to control the terms $E_{1}$ and $E_{2}$, we put $\left\|Z_{n}(f)\right\|_{\mathcal{F}}=\sup _{f \in \mathcal{F}}\left|Z_{n}(f)\right|$, and we use the following inequality due to Pisier: For all random variables $Z_{1}, Z_{2}, \ldots, Z_{N}$

$$
\mathbb{E}\left[\max _{1 \leq i \leq N}\left|Z_{i}\right|\right] \leq N^{\frac{1}{p}} \max _{1 \leq i \leq N}\left(\mathbb{E}\left|Z_{i}\right|^{p}\right)^{\frac{1}{p}} .
$$

Control of $E_{1}$ : For $f \in \mathcal{F}$, we write:

$$
f-\pi_{q_{0}}(f)=f-\pi_{q_{1}}(f)+\sum_{k=q_{0}+1}^{q_{1}}\left[\pi_{k}(f)-\pi_{k-1}(f)\right] .
$$

Therefore,

$$
\begin{aligned}
E_{1} & \equiv \mathbb{E}\left\|Z_{n}\left(f-\pi_{q_{0}}(f)\right)\right\|_{\mathcal{F}} \\
& \leq \mathbb{E}\left\|Z_{n}\left(f-\pi_{q_{1}}(f)\right)\right\|_{\mathcal{F}}+\sum_{k=q_{0}+1}^{q_{1}} \mathbb{E}\left\|Z_{n}\left(\pi_{k}(f)-\pi_{k-1}(f)\right)\right\|_{\mathcal{F}} \\
& \leq E_{1, q_{1}+1}+2 \sqrt{n} \sup _{f \in \mathcal{F}} \mathbb{E}\left|\Delta_{q_{1}}(f)\right|+\sum_{k=q_{0}+1}^{q_{1}} E_{1, k}
\end{aligned}
$$

where $E_{1, k}=\mathbb{E}\left\|Z_{n}\left(\pi_{k}(f)-\pi_{k-1}(f)\right)\right\|_{\mathcal{F}}, q_{0}+1 \leq k \leq q_{1}$ and $E_{1, q_{1}+1}=\mathbb{E}\left\|Z_{n}\left(\Delta_{q_{1}}(f)\right)\right\|_{\mathcal{F}}$. Note that $\pi_{k}(f)-$ $\pi_{k-1}(f)=\pi_{k}(f)-\pi_{k-1}\left(\pi_{k}(f)\right)$ and $\pi_{k}(f)$ take values on a finite set $N(k) \leq 2.2^{v k}$. Then using Pisier's inequality, we can write:

$$
E_{1, k} \leq 2^{\frac{v k}{p}} \max _{g \in \pi_{k}(\mathcal{F})}\left\|Z_{n}\left(g-\pi_{k-1}(g)\right)\right\|_{p} .
$$


Apply $H(p, X)$ to $h=g-\pi_{k-1}(g)$ to get:

$$
\begin{aligned}
\left\|Z_{n}(h)\right\|_{p} & \leq D^{\frac{1}{p}}\left(\|h\|_{v}^{p}+n^{1+\varepsilon-\frac{p}{2}}\|h\|_{r}^{p}\right)^{\frac{1}{p}} \\
& \leq D^{\frac{1}{p}}\left(\|h\|_{v}+n^{\frac{1+\varepsilon}{p}-\frac{1}{2}}\|h\|_{r}\right)
\end{aligned}
$$

Using the fact that

$$
\|X\|_{r} \leq\|X\|_{v}^{\frac{v}{r}} \times\|X\|_{\infty}^{\frac{r-v}{r}},
$$

we obtain

$$
\begin{aligned}
\left\|Z_{n}(h)\right\|_{p} & \leq D^{\frac{1}{p}} \cdot\left(2^{-(k-1)}+n^{\frac{1+\varepsilon}{p}-\frac{1}{2}} 2^{-\frac{(k-1) v}{r}}\right) \\
& \leq 2 D^{\frac{1}{p}} \cdot\left(2^{-k}+n^{\frac{1+\varepsilon}{p}-\frac{1}{2}} 2^{-\frac{k v}{r}}\right) .
\end{aligned}
$$

Hence,

$$
\begin{aligned}
E_{1, k} & \leq 2 D^{\frac{1}{p}} \cdot 2^{\frac{v k}{p}}\left(2^{-k}+n^{\frac{1+\varepsilon}{p}-\frac{1}{2}} 2^{-\frac{k v}{r}}\right) \\
& \leq 2 D^{\frac{1}{p}} \cdot\left(2^{-k\left(1-\frac{v}{p}\right)}+n^{\frac{1+\varepsilon}{p}-\frac{1}{2}} 2^{k\left(\frac{\nu}{p}-\frac{v}{r}\right)}\right) \\
& \leq C \cdot\left(2^{-k\left(1-\frac{v}{p}\right)}+n^{\frac{1+\varepsilon}{p}-\frac{1}{2}} 2^{k\left(\frac{v}{p}-\frac{v}{r}\right)}\right)
\end{aligned}
$$

Similarly for $E_{1, q_{1}+1}$ :

$$
E_{1, q_{1}+1} \leq C \cdot\left(2^{-\left(q_{1}+1\right)\left(1-\frac{v}{p}\right)}+n^{\frac{1+\varepsilon}{p}-\frac{1}{2}} 2^{\left(q_{1}+1\right)\left(\frac{v}{p}-\frac{v}{r}\right)}\right) .
$$

Finally, using that $\mathbb{E}\left|\Delta_{q_{1}}(f)\right|=\left\|\Delta_{q_{1}}(f)\right\|_{v}^{v} \leq 2^{-q_{1} v}$, we obtain:

$$
\begin{gathered}
E_{1} \leq C \cdot \sqrt{n} 2^{-q_{1} v}+\sum_{k=q_{0}+1}^{q_{1}+1} E_{1, k} \leq C \cdot \sqrt{n} 2^{-q_{1} v}+C \cdot \sum_{k=q_{0}+1}^{q_{1}+1}\left(2^{-k\left(1-\frac{v}{p}\right)}+n^{\frac{1+\varepsilon}{p}-\frac{1}{2}} 2^{k\left(\frac{v}{p}-\frac{v}{r}\right)}\right) \\
\leq C \cdot\left(\sqrt{n} 2^{-q_{1} v}+2^{-q_{0}\left(1-\frac{v}{p}\right)}+n^{\frac{1+\varepsilon}{p}-\frac{1}{2}}\left[2^{q_{1}\left(\frac{v}{p}-\frac{v}{r}\right)}-2^{q_{0}\left(\frac{v}{p}-\frac{v}{r}\right)}\right]\right) .
\end{gathered}
$$

Then,

$$
E_{1} \leq C \cdot\left(\sqrt{n} 2^{-q_{1} v}+2^{-q_{0}\left(1-\frac{v}{p}\right)}+n^{\frac{1+\varepsilon}{p}-\frac{1}{2}}\left[2^{q_{1}\left(\frac{v}{p}-\frac{v}{r}\right)}-2^{q_{0}\left(\frac{v}{p}-\frac{v}{r}\right)}\right]\right) .
$$

Control of $E_{2}$ : Noting that $|\Lambda| \leq 2 \times 2^{v q_{0}}$ (since if $F_{i, j} \neq \phi$, then $j=\{i-1, i, i+1\}$, because $\left|f_{x}-f_{x_{q_{0}}(i)}\right| \leq \Delta_{x_{q_{0}}(i)}$ and $\left.\left\|\Delta_{x_{q_{0}}(i)}\right\|_{v} \leq 2^{-q_{0}}\right)$ and $\left\|\phi_{i, j}-\psi_{i, j}\right\|_{\nu} \leq \delta$, using the inequality of Pisier, we get

$$
\begin{aligned}
E_{2} & =\mathbb{E}\left(\sup _{(i, j) \in \Lambda}\left|Z_{n}\left(\phi_{i, j}-\psi_{i, j}\right)\right|\right) \\
& \leq 2^{\frac{v q_{0}}{p}} \max _{(i, j) \in \Lambda}\left\|Z_{n}\left(\phi_{i, j}-\psi_{i, j}\right)\right\|_{p} .
\end{aligned}
$$

Again by $H(p, X)$,

$$
\begin{aligned}
E_{2} & \leq 2^{\frac{v q_{0}}{p}}\left[D^{\frac{1}{p}} \cdot\left(\left\|\phi_{i, j}-\psi_{i, j}\right\|_{v}+n^{\frac{1+\varepsilon}{p}-\frac{1}{2}}\left\|\phi_{i, j}-\psi_{i, j}\right\|_{r}\right)\right] \\
& \leq 2^{\frac{v q_{0}}{p}} D^{\frac{1}{p}} \cdot\left(\delta+n^{\frac{1+\varepsilon}{p}-\frac{1}{2}} \delta^{\frac{v}{r}}\right)
\end{aligned}
$$

Then,

$$
E_{2} \leq D^{\frac{1}{p}} \cdot 2^{\frac{v q_{0}}{p}}\left(\delta+n^{\frac{1+\varepsilon}{p}-\frac{1}{2}} \delta^{\frac{\nu}{r}}\right)
$$

Thus, from (2.3) and (2.4) we conclude that:

$$
W(n, \delta) \leq C \cdot\left[\sqrt{n} 2^{-q_{1} v}+2^{-q_{0}\left(1-\frac{v}{p}\right)}+2^{\frac{v q_{0}}{p}} \delta+n^{\frac{1+\varepsilon}{p}-\frac{1}{2}}\left(2^{q_{1}\left(\frac{v}{p}-\frac{v}{r}\right)}-2^{q_{0}\left(\frac{v}{p}-\frac{v}{r}\right)}+2^{\frac{v q_{0}}{p}} \delta^{\frac{v}{r}}\right)\right] .
$$


We have $\delta \sim 2^{-q_{0}}$ then $2^{\frac{v q_{0}}{p}} \cdot \delta \sim 2^{-q_{0}\left(1-\frac{\nu}{p}\right)} \sim \delta^{\left(1-\frac{v}{p}\right)}$, hence

$$
W(n, \delta) \leq C \cdot\left[\sqrt{n} 2^{-q_{1} v}+\delta^{\left(1-\frac{v}{p}\right)}+n^{\frac{1+\varepsilon}{p}-\frac{1}{2}} 2^{q_{1}\left(\frac{v}{p}-\frac{v}{r}\right)}\right] .
$$

Take $q_{1}$ such that $\sqrt{n} 2^{-q_{1} v} \sim n^{\frac{1+\varepsilon}{p}-\frac{1}{2}} 2^{q_{1}\left(\frac{v}{p}-\frac{v}{r}\right)}$ then

$$
2^{q_{1}} \sim n^{\frac{1-\frac{1+\varepsilon}{p}}{v\left(1+\frac{1}{p}-\frac{1}{r}\right)}} \Rightarrow q_{1} \sim \frac{\left(1-\frac{1+\varepsilon}{p}\right) \ln n}{v\left(1+\frac{1}{p}-\frac{1}{r}\right) \ln 2} .
$$

Therefore,

$$
W(n, \delta) \leq C \cdot\left(n^{-\frac{1}{2}+\frac{2+\varepsilon-\frac{p}{p}}{p+1-\frac{p}{T}}}+\delta^{\left(1-\frac{\nu}{p}\right)}\right) .
$$

As $q_{1}$ and $q_{0}$ have to satisfy $q_{0}<q_{1}$ then $\delta>n^{\frac{\frac{1+\varepsilon}{p}-1}{v\left(1+\frac{1}{p}-\frac{1}{r}\right)}}$. And to ensure that $W(n, \delta) \rightarrow_{\{n \rightarrow \infty, \delta \rightarrow 0\}} 0$, we need $-\frac{1}{2}+\frac{2+\varepsilon-\frac{p}{r}}{p+1-\frac{p}{r}}<0$ which is this

$$
\varepsilon<\frac{p}{2}\left(1-\frac{1}{p}+\frac{1}{r}\right)-1
$$

Proof of Remark 1. The proof of the first point of the Remark 1 has the same steps of the proof of Theorem 1 up to the inequality (2.2). This relation becomes in the case where $r=p$,

$$
\begin{aligned}
E_{1} & \leq C \cdot \sqrt{n} 2^{-q_{1} v}+C \cdot \sum_{k=q_{0}+1}^{q_{1}+1}\left(2^{-k\left(1-\frac{v}{p}\right)}+n^{\frac{1+\varepsilon}{p}-\frac{1}{2}}\right) \\
& \leq C \cdot\left(\sqrt{n} 2^{-q_{1} v}+2^{-q_{0}\left(1-\frac{v}{p}\right)}+q_{1} n^{\frac{1+\varepsilon}{p}-\frac{1}{2}}\right) .
\end{aligned}
$$

Since

$$
q_{1} \sim \frac{1}{v \ln 2}\left(1-\frac{1+\varepsilon}{p}\right) \ln n
$$

Therefore,

$$
W(n, \delta) \leq C \cdot\left(\ln n \cdot n^{-\frac{1}{2}+\frac{1+\varepsilon}{p}}+\delta^{\left(1-\frac{\nu}{p}\right)}\right) .
$$

\section{Limit Theorems for the Empirical VaR}

In this part we will apply the results of the previous section on the fluctuations of the empirical process to deduce asymptotic results on the $\widehat{\operatorname{VaR}}(q)$.

Theorem 2 Under conditions $H(X), H(F)$ and $H(p, X)$ where $\varepsilon<\frac{p}{2}\left(1-\frac{1}{p}+\frac{1}{r}\right)-1$, we have for $a_{n} \gg n^{-\frac{1}{2}}$,

$$
\left|\xi_{n}-\xi\right|=o_{p}\left(a_{n}\right)
$$

If in addition

$$
\sqrt{n}\left(F_{n}(\xi)-F(\xi)\right) \rightarrow^{d} \mathcal{N}\left(0, \sigma_{\infty}^{2}(\xi)\right)
$$

then

$$
\sqrt{n}\left(\xi_{n}-\xi\right) \longrightarrow^{d} \mathcal{N}\left(0, \frac{\sigma_{\infty}^{2}(\xi)}{f^{2}(\xi)}\right)
$$

The proof of the previous theorem is based on the two following lemmas:

Lemma 1 Under conditions $H(X), H(F)$ and $H(p, X)$ where $\varepsilon \leq \frac{p}{2}-1$, we have for $a_{n}>0$,

$$
\mathbb{P}\left(\left|\xi_{n}-\xi\right|>a_{n}\right) \leq C(\varepsilon, p, v, r, \xi) \cdot\left(n^{\frac{1}{2}} a_{n}\right)^{-p} .
$$

If in addition $a_{n} \gg n^{-\frac{1}{2}}$, then

$$
\left|\xi_{n}-\xi\right|=o_{p}\left(a_{n}\right)
$$


Proof of Lemma 1. Let $s=[n q]+1$. Then, we note that

$$
\begin{aligned}
\mathbb{P}\left(\xi_{n}<\xi-a_{n}\right) & =\mathbb{P}\left(s \text { or more of the } X_{i}(1 \leq i \leq n) \text { are }<\xi-a_{n}\right) \\
& =\mathbb{P}\left(\sum_{i=1}^{n} \mathbb{I}_{\left(X_{i}<\xi-a_{n}\right)} \geq s\right) \\
& =\mathbb{P}\left(F_{n}\left(\xi-a_{n}\right) \geq \frac{s}{n}\right) \\
& =\mathbb{P}\left(F_{n}\left(\xi-a_{n}\right)-F\left(\xi-a_{n}\right) \geq \frac{s}{n}-F\left(\xi-a_{n}\right)\right) .
\end{aligned}
$$

Since

$$
F_{n}\left(\xi_{n}\right)=\frac{s}{n}=F(\xi)+O\left(n^{-1}\right),(\text { see e.g. Sen, 1972) }
$$

then, using $H(F)$ and the first-order Taylor expansion of $F\left(\xi-a_{n}\right)$, one obtains

$$
\frac{s}{n}-F\left(\xi-a_{n}\right)=f(\xi) a_{n}[1+o(1)]
$$

Then

$$
\mathbb{P}\left(\xi_{n}<\xi-a_{n}\right)=\mathbb{P}\left(F_{n}\left(\xi-a_{n}\right)-F\left(\xi-a_{n}\right) \geq f(\xi) a_{n}[1+o(1)]\right) .
$$

And by Markov's inequality, this is bounded by

$$
\begin{aligned}
\mathbb{P}\left(\xi_{n}<\xi-a_{n}\right) & \leq\left(\frac{1}{f(\xi) a_{n}[1+o(1)]}\right)^{p} \mathbb{E}\left[F_{n}\left(\xi-a_{n}\right)-F\left(\xi-a_{n}\right)\right]^{p} \\
& \leq C \cdot\left(\frac{1}{f(\xi) a_{n}}\right)^{p} \mathbb{E}\left|F_{n}\left(\xi-a_{n}\right)-F\left(\xi-a_{n}\right)\right|^{p} .
\end{aligned}
$$

But,

$$
\mathbb{E}\left|F_{n}\left(\xi-a_{n}\right)-F\left(\xi-a_{n}\right)\right|^{p}=\left(\frac{1}{\sqrt{n}}\right)^{p} \mathbb{E}\left|Z_{n}\left(f_{\left(\xi-a_{n}\right)}\right)\right|^{p} .
$$

By $H(p, X)$,

$$
\begin{aligned}
\mathbb{E}\left|F_{n}\left(\xi-a_{n}\right)-F\left(\xi-a_{n}\right)\right|^{p} & \leq\left(\frac{1}{\sqrt{n}}\right)^{p} D \cdot\left(\left\|\mathbb{I}_{\left(X_{i}<\xi-a_{n}\right)}\right\|_{v}^{p}+n^{1+\varepsilon-\frac{p}{2}} \cdot\left\|\mathbb{I}_{\left(X_{i}<\xi-a_{n}\right)}\right\|_{r}^{p}\right) \\
& \leq n^{\frac{-p}{2}} D \cdot\left(F\left(\xi-a_{n}\right)^{\frac{p}{v}}+n^{1+\varepsilon-\frac{p}{2}} F\left(\xi-a_{n}\right)^{\frac{p}{r}}\right) .
\end{aligned}
$$

Then,

$$
\begin{aligned}
\mathbb{P}\left(\xi_{n}<\xi-a_{n}\right) & \leq C \cdot\left(\frac{1}{f(\xi) a_{n}}\right)^{p} n^{\frac{-p}{2}} D \cdot\left(F\left(\xi-a_{n}\right)^{\frac{p}{v}}+n^{1+\varepsilon-\frac{p}{2}} F\left(\xi-a_{n}\right)^{\frac{p}{r}}\right) \\
& \leq C \cdot D\left(\frac{1}{f(\xi)}\right)^{p} n^{\frac{-p}{2}} a_{n}^{-p}\left(F\left(\xi-a_{n}\right)^{\frac{p}{v}}+n^{1+\varepsilon-\frac{p}{2}} F\left(\xi-a_{n}\right)^{\frac{p}{r}}\right) \\
& \leq C(\varepsilon, p, v, r, \xi) \cdot\left(1+n^{1+\varepsilon-\frac{p}{2}}\right) n^{\frac{-p}{2}} a_{n}^{-p} .
\end{aligned}
$$

Consequently for $0<a_{n}$ and $\varepsilon \leq \frac{p}{2}-1$

$$
\mathbb{P}\left(\xi_{n}<\xi-a_{n}\right) \leq C(\varepsilon, p, v, r, \xi) \cdot n^{\frac{-p}{2}} a_{n}^{-p} .
$$

For the second term, we note that:

$$
\begin{aligned}
\mathbb{P}\left(\xi_{n}>\xi+a_{n}\right) & =\mathbb{P}\left(s \text { or less of the } X_{i}(1 \leq i \leq n) \text { are }<\xi+a_{n}\right) \\
& =\mathbb{P}\left(\sum_{i=1}^{n} \mathbb{I}_{\left(X_{i}<\xi+a_{n}\right)} \leq s\right) \\
& =\mathbb{P}\left(F_{n}\left(\xi+a_{n}\right) \leq \frac{s}{n}\right) \\
& =\mathbb{P}\left(F_{n}\left(\xi+a_{n}\right)-F\left(\xi+a_{n}\right) \leq \frac{s}{n}-F\left(\xi+a_{n}\right)\right) .
\end{aligned}
$$


But, using $H(F)$ and the first-order Taylor expansion of $F\left(\xi+a_{n}\right)$, one obtains

$$
\begin{aligned}
\mathbb{P}\left(\xi_{n}>\xi+a_{n}\right) & =\mathbb{P}\left(F_{n}\left(\xi+a_{n}\right)-F\left(\xi+a_{n}\right) \leq-f(\xi) a_{n}[1+o(1)]\right) \\
& =\mathbb{P}\left(F\left(\xi+a_{n}\right)-F_{n}\left(\xi+a_{n}\right) \geq f(\xi) a_{n}[1+o(1)]\right) .
\end{aligned}
$$

and by Markov's inequality, this is bounded by

$$
\begin{aligned}
\mathbb{P}\left(\xi_{n}>\xi+a_{n}\right) & \leq\left(\frac{1}{f(\xi) a_{n}[1+o(1)]}\right)^{p} \mathbb{E}\left[F\left(\xi+a_{n}\right)-F_{n}\left(\xi+a_{n}\right)\right]^{p}, \\
& \leq C \cdot\left(\frac{1}{f(\xi) a_{n}}\right)^{p} \mathbb{E}\left|F_{n}\left(\xi+a_{n}\right)-F\left(\xi+a_{n}\right)\right|^{p} .
\end{aligned}
$$

In the same way for the first term, we have

$$
\mathbb{E}\left|F_{n}\left(\xi+a_{n}\right)-F\left(\xi+a_{n}\right)\right|^{p}=\left(\frac{1}{\sqrt{n}}\right)^{p} \mathbb{E}\left|Z_{n}\left(f_{\left(\xi+a_{n}\right)}\right)\right|^{p} .
$$

By $H(p, X)$,

$$
\begin{aligned}
\mathbb{E}\left|F_{n}\left(\xi+a_{n}\right)-F\left(\xi+a_{n}\right)\right|^{p} & \leq\left(\frac{1}{\sqrt{n}}\right)^{p} D \cdot\left(\left\|\mathbb{I}_{\left(X_{i}<\xi+a_{n}\right)}\right\|_{v}^{p}+n^{1+\varepsilon-\frac{p}{2}} \cdot\left\|\mathbb{I}_{\left(X_{i}<\xi+a_{n}\right)}\right\|_{r}^{p}\right) \\
& \leq n^{\frac{-p}{2}} D \cdot\left(F\left(\xi+a_{n}\right)^{\frac{p}{v}}+n^{1+\varepsilon-\frac{p}{2}} F\left(\xi+a_{n}\right)^{\frac{p}{r}}\right) .
\end{aligned}
$$

Then,

$$
\begin{aligned}
\mathbb{P}\left(\xi_{n}>\xi+a_{n}\right) & \leq C \cdot\left(\frac{1}{f(\xi) a_{n}}\right)^{p} n^{\frac{-p}{2}} D \cdot\left(F\left(\xi+a_{n}\right)^{\frac{p}{v}}+n^{1+\varepsilon-\frac{p}{2}} F\left(\xi+a_{n}\right)^{\frac{p}{r}}\right) \\
& \leq C \cdot D \cdot\left(\frac{1}{f(\xi)}\right)^{p} n^{\frac{-p}{2}} a_{n}^{-p}\left(F\left(\xi+a_{n}\right)^{\frac{p}{v}}+n^{1+\varepsilon-\frac{p}{2}} F\left(\xi+a_{n}\right)^{\frac{p}{r}}\right) \\
& \leq C(\varepsilon, p, v, r, \xi) \cdot\left(1+n^{1+\varepsilon-\frac{p}{2}}\right) n^{\frac{-p}{2}} a_{n}^{-p} .
\end{aligned}
$$

Consequently for $0<a_{n}$ and $\varepsilon \leq \frac{p}{2}-1$

$$
\mathbb{P}\left(\xi_{n}>\xi+a_{n}\right) \leq C(\varepsilon, p, v, r, \xi) \cdot n^{\frac{-p}{2}} a_{n}^{-p} .
$$

Thus, from (3.1) and (3.2) we conclude for $0<a_{n}$ and $\varepsilon \leq \frac{p}{2}-1$

$$
\mathbb{P}\left(\left|\xi_{n}-\xi\right|>a_{n}\right) \leq C(\varepsilon, p, v, r, \xi) \cdot\left(n^{\frac{1}{2}} a_{n}\right)^{-p} .
$$

Finally, if $a_{n} \gg n^{-\frac{1}{2}}$, then

$$
\mathbb{P}\left(\left|\xi_{n}-\xi\right|>a_{n}\right) \rightarrow_{n \rightarrow \infty} 0 .
$$

The following lemma studies the proximity between $Z_{n}\left(f_{\xi_{n}}\right)=\sqrt{n}\left(F_{n}\left(\xi_{n}\right)-F\left(\xi_{n}\right)\right)$ and $Z_{n}\left(f_{\xi}\right)=\sqrt{n}\left(F_{n}(\xi)-\right.$ $F(\xi))$.

Lemma 2 Under conditions $H(X), H(F)$ and $H(p, X)$ where $\varepsilon<\frac{p}{2}\left(1-\frac{1}{p}+\frac{1}{r}\right)-1$, we have for $a_{n} \gg n^{-\frac{1}{2}}$ and $b_{n} \gg \max \left(n^{-\frac{1}{2}+\frac{2+\varepsilon-\frac{p}{p}}{p+1-\frac{p}{r}}}, a_{n}^{\left(\frac{1}{p}-\frac{1}{p}\right)}\right)$,

$$
\left|\sqrt{n}\left(F_{n}\left(\xi_{n}\right)-F\left(\xi_{n}\right)\right)-\sqrt{n}\left(F_{n}(\xi)-F(\xi)\right)\right|=o_{p}\left(b_{n}\right) .
$$

Proof of Lemma 2. Let $0<a_{n}$ and $0<b_{n}$, we note that

$$
\begin{aligned}
\mathbb{P}\left(\left|Z_{n}\left(f_{\xi_{n}}-f_{\xi}\right)\right|>b_{n}\right) & =\mathbb{P}\left(\left|Z_{n}\left(f_{\xi_{n}}-f_{\xi}\right)\right|>b_{n} \cap\left|\xi_{n}-\xi\right| \leq a_{n}\right)+\mathbb{P}\left(\left|Z_{n}\left(f_{\xi_{n}}-f_{\xi}\right)\right|>b_{n} \cap\left|\xi_{n}-\xi\right|>a_{n}\right) \\
& \leq \mathbb{P}\left(\left|Z_{n}\left(f_{\xi_{n}}-f_{\xi}\right)\right| \mathbb{I}_{\left(\xi_{n}-\xi \mid \leq a_{n}\right)}>b_{n}\right)+\mathbb{P}\left(\left|\xi_{n}-\xi\right|>a_{n}\right) .
\end{aligned}
$$


If $H(p, X)$ is verified for $\varepsilon<\frac{p}{2}\left(1-\frac{1}{p}+\frac{1}{r}\right)-1 \leq \frac{p}{2}-1$ and $0<a_{n}$, then by Lemma 1 :

$$
\mathbb{P}\left(\left|\xi_{n}-\xi\right|>a_{n}\right) \leq C \cdot\left(n^{\frac{1}{2}} a_{n}\right)^{-p} .
$$

If $H(F)$ is verified, then $F$ is locally Lipschitz, then for $|y-\xi| \leq a_{n}$, we have

$$
\left\|f_{y}-f_{\xi}\right\|_{v}=|F(y)-F(\xi)|^{\frac{1}{v}} \leq C(v, \xi) \cdot|y-\xi|^{\frac{1}{v}} \leq C(v, \xi) \cdot a_{n}^{\frac{1}{v}} .
$$

In addition, by Markov's inequality and Theorem 1 for $a_{n}>\frac{1}{C(v, \xi)} n^{\frac{\frac{1+\varepsilon}{p}-1}{1+\frac{1}{p}-\frac{1}{r}}}$

$$
\begin{aligned}
\mathbb{P}\left(\left|Z_{n}\left(f_{\xi_{n}}-f_{\xi}\right)\right| \mathbb{I}_{\left(\left|\xi_{n}-\xi\right| \leq a_{n}\right)}>b_{n}\right) & \leq \frac{1}{b_{n}} \mathbb{E}\left|\sup _{|y-\xi| \leq a_{n}}\right| Z_{n}\left(f_{y}-f_{\xi}\right) \mid \\
& \leq C \cdot b_{n}^{-1}\left(n^{-\frac{1}{2}+\frac{2+\varepsilon-\frac{p}{p}}{p+1-\frac{1}{r}}}+C \cdot a_{n}^{\left(\frac{1}{\nu}-\frac{1}{p}\right)}\right) .
\end{aligned}
$$

Consequently, for $a_{n}>\frac{1}{C(v, \xi)} n^{\frac{\frac{1+\varepsilon}{p}-1}{1+\frac{1}{p}-\frac{1}{r}}}$ where $\varepsilon<\frac{p}{2}\left(1-\frac{1}{p}+\frac{1}{r}\right)-1$ and $0<b_{n}$

$$
\mathbb{P}\left(\left|Z_{n}\left(f_{\xi_{n}}-f_{\xi}\right)\right|>b_{n}\right) \leq C \cdot\left[b_{n}^{-1}\left(n^{-\frac{1}{2}+\frac{2+\varepsilon-\frac{p}{p}}{p+1-\frac{1}{r}}}+C \cdot a_{n}^{\left(\frac{1}{\bar{\nu}}-\frac{1}{p}\right)}\right)+\left(n^{\frac{1}{2}} a_{n}\right)^{-p}\right] .
$$

If $a_{n} \gg n^{-\frac{1}{2}}$ and $b_{n} \gg \max \left(n^{-\frac{1}{2}+\frac{2+\varepsilon-\frac{p}{p}}{p+1-\frac{p}{r}}}, a_{n}^{\left(\frac{1}{v}-\frac{1}{p}\right)}\right)$, then

$$
\left|Z_{n}\left(f_{\xi_{n}}-f_{\xi}\right)\right|=o_{p}\left(b_{n}\right) .
$$

Finally, by the definition of $Z_{n}\left(f_{x}\right)$, we obtain

$$
\left|\sqrt{n}\left(F_{n}\left(\xi_{n}\right)-F\left(\xi_{n}\right)\right)-\sqrt{n}\left(F_{n}(\xi)-F(\xi)\right)\right|=o_{p}\left(b_{n}\right) .
$$

Proof of Theorem 2. By Lemmas 1 and 2 for $a_{n} \gg n^{-\frac{1}{2}}$ and $b_{n} \gg \max \left(n^{-\frac{1}{2}+\frac{2+\varepsilon-\frac{p}{p}}{p+1-\frac{p}{T}}}, a_{n}^{\left(\frac{1}{\nu}-\frac{1}{p}\right)}\right)$, we have

$$
\left|\sqrt{n}\left(F_{n}\left(\xi_{n}\right)-F\left(\xi_{n}\right)\right)-\sqrt{n}\left(F_{n}(\xi)-F(\xi)\right)\right|=o_{p}\left(b_{n}\right) .
$$

Since

$$
F_{n}\left(\xi_{n}\right)=\frac{s}{n}=F(\xi)+O\left(n^{-1}\right),
$$

then,

$$
\sqrt{n}\left(F_{n}\left(\xi_{n}\right)-F\left(\xi_{n}\right)\right)=\sqrt{n}\left(F(\xi)-F\left(\xi_{n}\right)\right)+O\left(n^{-\frac{1}{2}}\right) .
$$

If $H(F)$ is satisfied, then by the Mean Value Theorem of $F(\xi)-F\left(\xi_{n}\right)$,

$$
F(\xi)-F\left(\xi_{n}\right)=\left(\xi-\xi_{n}\right) f\left(\theta \xi_{n}+(1-\theta) \xi\right)
$$

where $\theta \in[0,1]$. Then

$$
\sqrt{n}\left(F_{n}\left(\xi_{n}\right)-F\left(\xi_{n}\right)\right)=\sqrt{n}\left(\xi-\xi_{n}\right) f\left(\theta \xi_{n}+(1-\theta) \xi\right)+O\left(n^{-\frac{1}{2}}\right) .
$$

Hence,

$$
\left|\sqrt{n}\left(\xi-\xi_{n}\right) f\left(\theta \xi_{n}+(1-\theta) \xi\right)+O\left(n^{-\frac{1}{2}}\right)-\sqrt{n}\left(F_{n}(\xi)-F(\xi)\right)\right|=o_{p}\left(b_{n}\right)
$$

But we have,

$$
\sqrt{n}\left(F_{n}(\xi)-F(\xi)\right) \rightarrow^{d} \mathcal{N}\left(0, \sigma_{\infty}^{2}(\xi)\right)
$$

And by Lemma 1 for $a_{n} \gg n^{-\frac{1}{2}}$,

$$
f\left(\theta \xi_{n}+(1-\theta) \xi\right)=\left[f\left(\xi+o_{p}\left(a_{n}\right)\right)\right] \rightarrow_{n \rightarrow \infty} f(\xi) \text { in probability. }
$$


Then by (3.3), (3.4), (3.5), (3.6) and Slutsky's Theorem (Cramér, 1946, p. 254), we have:

$$
\sqrt{n}\left(f(\xi)\left(\xi-\xi_{n}\right)\right) \rightarrow^{d} \mathcal{N}\left(0, \sigma_{\infty}^{2}(\xi)\right) .
$$

Which is equivalent in the result to,

$$
\sqrt{n}\left(\xi_{n}-\xi\right) \longrightarrow^{d} \mathcal{N}\left(0, \frac{\sigma_{\infty}^{2}(\xi)}{f^{2}(\xi)}\right)
$$

\section{Applications}

In this section we apply the previous results for different sequences. Using the findings of $\mathrm{Hu}(2003$, p. 1124) and Peligrad (1985, Theorem 2.1, p. 1305), we apply our result to $\varphi$-mixing case. Making use of the result of Utev and Peligrad (2003, Theorem 2.1 and 2.2), we apply our result to the $\rho$-mixing case and to $\alpha$-mixing by mean of the results in Shao and Yu (1996, Theorem 4.1) and Rio (1997, Theorem 7.2). We also consider the nonlinear functional of Gaussian sequences to which we apply the result of Ben Hariz (2011) and Breuer and Major (1983). Finally we compare the results with those in the existing literature.

4.1 -mixing Process

Corollary 1 Under condition $H(X)$, if the $\varphi$-mixing coefficient satisfies

$$
\sum_{i=0}^{\infty} \varphi^{\frac{1}{p}}\left(2^{i}\right)<\infty \quad \text { with } p>2
$$

Then, for $\delta>n^{-\frac{1}{2}\left(1-\frac{1}{p}\right)}$, there is a positive constant $C(p, \varphi()$.$) such that for any f \in \mathcal{F}$

$$
\mathbb{E}\left[\sup _{\left\|f_{x}-f_{y}\right\|_{2} \leq \delta}\left|Z_{n}\left(f_{x}-f_{y}\right)\right|\right] \leq C(p, \varphi(.)) \cdot\left(\ln n \cdot n^{\frac{1}{p}-\frac{1}{2}}+\delta^{\left(1-\frac{2}{p}\right)}\right) .
$$

If $H(F)$ is verified, then for $a_{n} \gg n^{-\frac{1}{2}}$ we have

$$
\left|\xi_{n}-\xi\right|=o_{p}\left(a_{n}\right)
$$

and if in addition $0<\sigma_{\infty}^{2}<\infty$, then

$$
\sqrt{n}\left(\xi_{n}-\xi\right) \longrightarrow^{d} \mathcal{N}\left(0, \frac{\sigma_{\infty}^{2}(\xi)}{f^{2}(\xi)}\right)
$$

Proof of Corollary 1. When $\left(X_{i}\right)_{i \geq 1}$ are identically distributed, using a Lemma by Hu (2003, p. 1124), if

$$
\sum_{i=0}^{\infty} \varphi^{\frac{1}{p}}\left(2^{i}\right)<\infty
$$

then, there exists a positive constant $K=K(p, \varphi()$.$) such that for all n \geq 1$ and for any $f$

$$
\mathbb{E}\left|Z_{n}(f)\right|^{p} \leq C(p, \varphi(\cdot)) \cdot\left(\|f\|_{2}^{p}+n^{1-\frac{p}{2}}\|f\|_{p}^{p}\right) .
$$

Then $H(p, X)$ is satisfied with $\varepsilon=0, v=2$ and $p=r$. Apply now Theorem 1 for $\delta>n^{-\frac{1}{2}\left(1-\frac{1}{p}\right)}$, to obtain

$$
\mathbb{E}\left[\sup _{\left\|f_{x}-f_{y}\right\|_{2} \leq \delta}\left|Z_{n}\left(f_{x}-f_{y}\right)\right|\right] \leq C \cdot\left(\ln n \cdot n^{\frac{1}{p}-\frac{1}{2}}+\delta^{\left(1-\frac{2}{p}\right)}\right) .
$$

If $H(F)$ is verified and $a_{n} \gg n^{-\frac{1}{2}}$, then by Lemma 1 for $p>2$ we obtain

$$
\left|\xi_{n}-\xi\right|=o_{p}\left(a_{n}\right)
$$

To show that

$$
\sqrt{n}\left(F_{n}(\xi)-F(\xi)\right) \rightarrow^{d} \mathcal{N}\left(0, \sigma_{\infty}^{2}(\xi)\right)
$$


we will apply a result by Peligrad (1985, Theorem 2.1, p. 1305) with $Y_{i} \equiv \mathbb{I}_{\left(X_{i} \leq \xi\right)}-F(\xi), \sigma_{n}^{2}=\mathbb{E}\left[\sum_{i=1}^{n} Y_{i}\right]^{2}$ and $W_{n}(t):=\frac{1}{\sigma_{n}} \sum_{i=1}^{[n t]} Y_{i}, t \in[0,1]$ and $0<\sigma_{\infty}^{2}<\infty$. If we have $0<\sigma_{\infty}^{2}<\infty$, then $\frac{\sigma_{n}^{2}}{n} \rightarrow_{n \rightarrow \infty} \sigma_{\infty}^{2}$.

The condition (L) therein can be written for $\epsilon>0$,

$$
\begin{aligned}
\frac{1}{\sigma_{n}^{2}} \sum_{i=1}^{n} \mathbb{E}\left[Y_{i}^{2} \mathbb{I}_{\left[Y_{i}^{2}>\epsilon \sigma_{n}^{2}\right]}\right] & \leq \frac{n}{\sigma_{n}^{2}} \mathbb{E}\left[Y_{i}^{2} \mathbb{I}_{\left[Y_{i}^{2}>\epsilon \sigma_{n}^{2}\right]}\right] \\
& \leq \frac{C \cdot n}{\sigma_{n}^{2}} \mathbb{P}\left[\left[\mathbb{I}_{\left(X_{i} \leq \xi\right)}-F(\xi)\right]^{2}>\epsilon \sigma_{n}^{2}\right] \\
& \leq \frac{C \cdot n}{\epsilon \sigma_{n}^{4}} \mathbb{E}\left[\left[\mathbb{I}_{\left(X_{i} \leq \xi\right)}-F(\xi)\right]^{2}\right] \rightarrow_{n \rightarrow \infty} 0 .
\end{aligned}
$$

The conditions:

(A) $\sigma_{n}^{2}=n h(n)$ où $h(n)$ is a slowly varying function defined on $\mathbb{R}$,

(B) $\sup _{m \geq 0, n \geq 1}\left[\mathbb{E}\left(\sum_{i=1}^{m+n} Y_{i}-\sum_{i=1}^{m} Y_{i}\right)^{2} / \sigma_{n}^{2}\right]<\infty$,

therein are a result of $0<\sigma_{\infty}^{2}<\infty$. We take $t=1$ to conclude

$$
\sqrt{n}\left(F_{n}(\xi)-F(\xi)\right) \rightarrow^{d} \mathcal{N}\left(0, \sigma_{\infty}^{2}(\xi)\right) .
$$

Therefore by Theorem 2

$4.2 \rho$-mixing Process

$$
\sqrt{n}\left(\xi_{n}-\xi\right) \longrightarrow^{d} \mathcal{N}\left(0, \frac{\sigma_{\infty}^{2}(\xi)}{f^{2}(\xi)}\right)
$$

For a stationary sequence $\left(X_{i}\right)_{i \in \mathbb{Z}}$, we define

$$
\begin{aligned}
\alpha_{n}^{*} & =\sup _{S, T \subset \mathbb{Z}, \text { dist }(S, T) \geq n} \alpha\left(\mathcal{M}_{T}, \mathcal{M}_{S}\right), \\
\rho_{n}^{*} & =\sup _{S, T \subset \mathbb{Z}, \operatorname{dist}(S, T) \geq n} \rho\left(\mathcal{M}_{T}, \mathcal{M}_{S}\right),
\end{aligned}
$$

where $\mathcal{M}_{T}=\sigma\left(X_{i}, i \in T\right), \mathcal{M}_{S}=\sigma\left(X_{i}, i \in S\right)$. We apply a result by Utev and Peligrad (2003, Theorems 2.1 and 2.2) to prove the following Theorems:

Corollary 2 Under condition $H(X)$, we assume: $H(\rho)$ : There exists a real number $0 \leq \eta<1$ and integer number $N \geq 1$ such that $\rho_{N}^{*} \leq \eta$. Then, for $p>2$ and $\delta>n^{-\frac{1}{2}\left(1-\frac{1}{p}\right)}$, there is a positive constant $C(p, N, \eta)$ such that for any $f \in \mathcal{F}$

$$
\mathbb{E}\left[\sup _{\left\|f_{x}-f_{y}\right\|_{2} \leq \delta}\left|Z_{n}\left(f_{x}-f_{y}\right)\right|\right] \leq C(p, N, \eta) \cdot\left(\ln n \cdot n^{\frac{1}{p}-\frac{1}{2}}+\delta^{\left(1-\frac{2}{p}\right)}\right) .
$$

If $H(F)$ is verified, then for $a_{n} \gg n^{-\frac{1}{2}}$ we have

$$
\left|\xi_{n}-\xi\right|=o_{p}\left(a_{n}\right) \quad \text { in probability. }
$$

If in addition the sequence $\left(X_{i}\right)_{i \geq 1}$ is stongly mixing and $0<\sigma_{\infty}^{2}<\infty$, then

$$
\sqrt{n}\left(\xi_{n}-\xi\right) \longrightarrow^{d} \mathcal{N}\left(0, \frac{\sigma_{\infty}^{2}(\xi)}{f^{2}(\xi)}\right) .
$$

Proof of Corollary 2. Assuming that the condition $H(\rho)$ is satisfied and the random variables are identically distributed, then by Utev and Peligrad (2003, Theorem 2.1), for any $p>2$, there exists a positive constant $D=$ $D(p, N, \eta)$ such that for $n \geq 1$,

$$
\mathbb{E}\left|Z_{n}(f)\right|^{p} \leq D\left(\|f\|_{2}^{p}+n^{1-\frac{p}{2}}\|f\|_{p}^{p}\right) .
$$

Apply now Theorem 1 with the condition $H(p, X)$ where $\varepsilon=0, v=2$ and $p=r$, we obtain

$$
\mathbb{E}\left[\sup _{\left\|f_{x}-f_{y}\right\|_{2} \leq \delta}\left|Z_{n}\left(f_{x}-f_{y}\right)\right|\right] \leq C \cdot\left(\ln n \cdot n^{\frac{1}{p}-\frac{1}{2}}+\delta^{\left(1-\frac{2}{p}\right)}\right) .
$$


If $H(F)$ is verified and $a_{n} \gg n^{-\frac{1}{2}}$, then by Lemma 1 for $p>2$ we obtain

$$
\left|\xi_{n}-\xi\right|=o_{p}\left(a_{n}\right) \quad \text { in probability. }
$$

To show that

$$
\sqrt{n}\left(F_{n}(\xi)-F(\xi)\right) \rightarrow^{d} \mathcal{N}\left(0, \sigma_{\infty}^{2}(\xi)\right),
$$

we will apply a result by Utev and Peligrad (2003, Theorem 2.2, p. 105) with $\xi_{n i} \equiv \mathbb{I}_{\left(X_{i} \leq \xi\right)}-F(\xi), \sigma_{n}^{2}=\mathbb{E}\left[\sum_{i=1}^{n} \xi_{n i}\right]^{2}$, $k_{n}=n$ and $W_{n}(t):=\frac{1}{\sigma_{n}} \sum_{i=1}^{v_{t}} \xi_{n i}$ where $v_{t}=[n t]$ and $t \in[0,1]$. Si on a $0<\sigma_{\infty}^{2}<\infty$, alors $\frac{\sigma_{n}^{2}}{n} \rightarrow_{n \rightarrow \infty} \sigma_{\infty}^{2}$. The condition (2.5) of Utev and Peligrad (2003):

(2.5) $\lim _{n \rightarrow \infty} \sup \left[n \mathbb{E}\left(\xi_{n 1}\right)^{2} / \sigma_{n}^{2}\right] \leq C$, is a consequence of $0<\sigma_{\infty}^{2}<\infty$. The condition (2.3) is proved in Corollary 2: (condition (L). We take $t=1$ to conclude

$$
\sqrt{n}\left(F_{n}(\xi)-F(\xi)\right) \rightarrow^{d} \mathcal{N}\left(0, \sigma_{\infty}^{2}(\xi)\right) .
$$

Therefore by Theorem 2

$$
\sqrt{n}\left(\xi_{n}-\xi\right) \longrightarrow^{d} \mathcal{N}\left(0, \frac{\sigma_{\infty}^{2}(\xi)}{f^{2}(\xi)}\right)
$$

$4.3 \alpha$-mixing Process

Corollary 3 Under conditions $H(X)$ and $H(F)$, if the $\alpha$-mixing coefficient satisfies

$$
\alpha(n) \leq C n^{-\theta} \text { for some } C \geq 1 \text { and } \theta>1+\sqrt{2} \text {. }
$$

Then, for $a_{n} \gg n^{-\frac{1}{2}}$ we have

$$
\left|\xi_{n}-\xi\right|=o_{p}\left(a_{n}\right)
$$

and if in addition $0<\sigma_{\infty}^{2}<\infty$, then

$$
\sqrt{n}\left(\xi_{n}-\xi\right) \longrightarrow^{d} \mathcal{N}\left(0, \frac{\sigma_{\infty}^{2}(\xi)}{f^{2}(\xi)}\right)
$$

Proof of Corollary 3. When $\left(X_{i}\right)_{i \geq 1}$ are identically distributed, then by Shao and Yu (1996, Theorem 4.1), if

$$
\alpha(n) \leq C n^{-\theta} \text { for } C>0 \text { and } \theta>0 .
$$

Then, for some real numbers $2<p<r \leq \infty, 2<v \leq r, \varepsilon>0, \theta>\frac{v}{v-2}$ and $\theta \geq \frac{(p-1) r}{r-p}$, there is a constant $K=K(v, p, r, \varepsilon, \theta, C)<\infty$ such that for any $f \in \mathcal{F}$

$$
\mathbb{E}\left|Z_{n}(f)\right|^{p} \leq K\left(\|f\|_{v}^{p}+n^{1+\varepsilon-\frac{p}{2}}\|f\|_{r}^{p}\right)
$$

which satisfies $H(p, X)$. If $\varepsilon \leq \frac{p}{2}-1$ and $a_{n} \gg n^{-\frac{1}{2}}$, then by Lemma 1 we have

$$
\left|\xi_{n}-\xi\right|=o_{p}\left(a_{n}\right) \text {. }
$$

For determining $\theta$ which allows to apply Theorem 1 we need $v<p<r$ and $\frac{p}{2}\left(1-\frac{1}{p}+\frac{1}{r}\right)-1>0$. Now we have

$$
\theta \geq \frac{(p-1) r}{r-p} \Leftrightarrow p \leq \frac{r(\theta+1)}{\theta+r},
$$

and

$$
\theta>\frac{v}{v-2} \Leftrightarrow v>\frac{2 \theta}{\theta-1} .
$$

Since $v<p$ we need

$$
\frac{2 \theta}{\theta-1}<\frac{r(\theta+1)}{\theta+r}
$$

which is satisfied if

$$
\theta>1+\sqrt{2}\left(\frac{\sqrt{r(r-1)}+\sqrt{2}}{r-2}\right) .
$$


Consequently, we take $\theta=1+\eta$ where $\eta>\sqrt{2}$. For $\eta>\sqrt{2}$ we have $2+\frac{2}{\eta}<2+\frac{2 \eta^{2}+6 \eta+4}{\eta^{3}+\eta^{2}+2 \eta+4}<2+\frac{2(2 \eta+3)}{\eta^{2}-2}$, then we choose $v, p, r$
i) $v=2+\frac{2}{\eta}$,
ii) $p=2+\frac{2 \eta^{2}+6 \eta+4}{\eta^{3}+\eta^{2}+2 \eta+4}$,
iii) $r=2+\frac{2(2 \eta+3)}{\eta^{2}-2}$.

With these choices we have $v<p<r$ and

$$
\frac{p}{2}\left(1-\frac{1}{p}+\frac{1}{r}\right)-1>0
$$

Then we have

$$
W\left(n, a_{n}\right) \leq C .\left(n^{-\frac{1}{2}+\frac{2+\varepsilon-\frac{p}{T}}{p+1-\frac{p}{r}}}+a_{n}^{\frac{1}{v}-\frac{1}{p}}\right) \rightarrow_{n \rightarrow \infty} 0 .
$$

If in addition $0<\sigma_{\infty}^{2}<\infty$, then by Rio (1997, Theorem 7.2) for

$$
\alpha(n) \leq C n^{-\theta} \text { where } C \geq 1 \text { and } \theta>1,
$$

we have

$$
\sqrt{n}\left(F_{n}(\xi)-F(\xi)\right) \rightarrow^{d} \mathcal{N}\left(0, \sigma_{\infty}^{2}(\xi)\right) .
$$

Finally, by applying Theorem 2 for $a_{n} \gg n^{-\frac{1}{2}}$, we obtain that

$$
\sqrt{n}\left(\xi_{n}-\xi\right) \longrightarrow^{d} \mathcal{N}\left(0, \frac{\sigma_{\infty}^{2}(\xi)}{f^{2}(\xi)}\right) .
$$

4.4 Nonlinear Functional of Gaussian Sequences

Corollary 4 Let $X_{i}=G\left(Z_{i}\right)$ where $G$ is a measurable function and $\left(Z_{i}\right)$ is a stationary Gaussian sequence with zero mean and covariance function

$$
\varrho(n)=E\left(Z_{i} Z_{i+n}\right) .
$$

Assume $\sum_{i=0}^{\infty}\left\lfloor\varrho(i) \mid<\infty\right.$. Then, for $p>2$ and $\delta>n^{-\frac{1}{2}\left(1-\frac{1}{p}\right)}$, there is a positive constant $C(p, \varrho)$ such that for any $f \in \mathcal{F}$

$$
\mathbb{E}\left[\sup _{\left\|f_{x}-f_{y}\right\|_{2} \leq \delta}\left|Z_{n}\left(f_{x}-f_{y}\right)\right|\right] \leq C(p, \varrho) \cdot\left(\ln n \cdot n^{\frac{1}{p}-\frac{1}{2}}+\delta^{\left(1-\frac{2}{p}\right)}\right) .
$$

If $H(F)$ is verified, then for $a_{n} \gg n^{-\frac{1}{2}}$ we have

$$
\left|\xi_{n}-\xi\right|=o_{p}\left(a_{n}\right) \quad \text { in probability }
$$

and if in addition $0<\sigma_{\infty}^{2}<\infty$, then

$$
\sqrt{n}\left(\xi_{n}-\xi\right) \longrightarrow^{d} \mathcal{N}\left(0, \frac{\sigma_{\infty}^{2}(\xi)}{f^{2}(\xi)}\right)
$$

Proof of Corollary 4. The proof of this corollary is a consequence of the following results:

Lemma 3 (Ben Hariz, 2011) Let $p$ be an even integer and assume that $\sum_{i=0}^{\infty}|\varrho(i)|<\infty$, then there exists a constant $K=K(p, \varrho)$ such that for all $n>0$,

$$
\mathbb{E}\left(\frac{1}{\sqrt{n}} \sum_{i=1}^{n} f\left(Z_{i}\right)-E\left(f\left(Z_{i}\right)\right)\right)^{p} \leq K(p, \varrho)\left(\|f\|_{2}^{p}+n^{1-\frac{p}{2}}\|f\|_{p}^{p}\right) .
$$

We apply Lemma 3 for $f(Z)=\mathbb{I}_{G(Z) \leq x}$. Then $H(p, X)$ is satisfied with $\varepsilon=0, v=2$ and $p=r$. If $H(F)$ is verified, then by Lemma 1 for $a_{n} \gg n^{-\frac{1}{2}}$, we have

$$
\left|\xi_{n}-\xi\right|=o_{p}\left(a_{n}\right) .
$$


And by Theorem 1

$$
W(n, \delta) \leq C .\left(\ln n \cdot n^{-\frac{1}{2}+\frac{1}{p}}+\delta^{\left(1-\frac{2}{p}\right)}\right) .
$$

For the central limit theorem we need to apply the following results due to Breuer and Major (1983), (see also Csörgo, Sándor \& Mielniczuk, 1996, for a functional extension) .

Lemma 4 Let $\left(Z_{i}\right)$ be a stationary Gaussian sequence with a covariance function satisfying $\sum_{i=0}^{\infty}|\varrho(i)|<\infty$, then

$$
\frac{1}{\sqrt{n}} \sum_{i=1}^{n}\left(\mathbb{I}_{G\left(Z_{i}\right) \leq x}-F(x)\right) \longrightarrow^{d} \mathcal{N}\left(0, \sigma_{\infty}^{2}(x)\right)
$$

where $\sigma_{n}^{2}(x)=\operatorname{Var}\left(\mathbb{I}_{G\left(Z_{i}\right) \leq x}\right)+2 \sum_{i=1}^{\infty} \operatorname{Cov}\left(\mathbb{I}_{G\left(Z_{1}\right) \leq x}, \mathbb{I}_{G\left(Z_{i}\right) \leq x}\right)$.

If $0<\sigma_{\infty}^{2}<\infty$, then by Lemma 3, Lemma 4 and Theorem 2 we have

$$
\sqrt{n}\left(\xi_{n}-\xi\right) \longrightarrow^{d} \mathcal{N}\left(0, \frac{\sigma_{\infty}^{2}(\xi)}{f^{2}(\xi)}\right) .
$$

\subsection{Comparison with the Existing Results of the Literature}

- In Sen (1972), Sen has proved that for a $\varphi$-mixing sequence of random variables, if we have

$$
\sum_{i=0}^{\infty} \varphi^{\frac{1}{2}}(i)<\infty
$$

then

$$
\sqrt{n}\left(\xi_{n}-\xi\right) \longrightarrow^{d} \mathcal{N}\left(0, \frac{\sigma_{\infty}^{2}(\xi)}{f^{2}(\xi)}\right)
$$

which is stronger than our condition:

$$
\sum_{i=0}^{\infty} \varphi^{\frac{1}{p}}\left(2^{i}\right)<\infty
$$

Indeed, $\sum_{i=0}^{\infty} \varphi^{\frac{1}{2}}(i)<\infty$ needs an algebraic decay of the the mixing coefficient $\varphi(i)$, and $\sum_{i=0}^{\infty} \varphi^{\frac{1}{p}}\left(2^{i}\right)<\infty$ needs only a logarithmic decay.

- In 2005, Chen and Tang studied the nonparametric estimation of the Value at Risk (VaR) for a geometric $\alpha$-mixing sequence of random variables, that means

$$
\alpha(k) \leq c \rho^{k} \text { where } k \geq 1, c>0 \text { and } \rho \in(0,1) .
$$

Using the kernel estimation of the VaR:

$$
\widehat{F}_{n, h}\left(\widehat{\operatorname{VaR}}_{h}(q)\right)=\frac{1}{n} \sum_{i=1}^{n} G\left(\frac{\widehat{\operatorname{VaR}}_{h}(q)-X_{i}}{h}\right)=q,
$$

where $G(x)=\int_{-\infty}^{x} K(u) d(u)$ is a distribution function of a kernel density $K$, they showed that:

$$
\begin{gathered}
\left|\widehat{\operatorname{VaR}}_{h}(q)-\operatorname{VaR}(q)\right|=o_{a . s .}\left(n^{-\frac{1}{2}} \ln (n)\right) . \\
\sqrt{n}\left(\widehat{\operatorname{VaR}}_{h}(q)-\operatorname{VaR}(q)\right) \rightarrow{ }^{d} \mathcal{N}\left(0, \frac{\sigma_{\infty}^{2}(\operatorname{VaR}(q))}{f^{2}(\operatorname{VaR}(q))}\right) .
\end{gathered}
$$

- Lahiri and Sun (2009) showed that for a $\alpha$-mixing sequence of random variables such that

$$
\alpha(n) \leq d n^{-\theta} \text { where } \theta>12,
$$

the empirical $\widehat{\operatorname{VaR}}(q)$ satisfy for a constant $C>0$ and $n \geq 1$

$$
\sup _{x \in \mathbb{R}}\left|\mathbb{P}\left[\sqrt{n}\left(\xi_{n}-\xi\right) \leq x\right]-\Phi\left[x \times \frac{f(\xi)}{\sigma_{\infty}(\xi)}\right]\right| \leq \frac{C}{\sqrt{n}},
$$


where $\Phi$ is the standard normal distribution. In particular they obtained as $n \rightarrow \infty$,

$$
\sqrt{n}\left(\xi_{n}-\xi\right) \longrightarrow^{d} \mathcal{N}\left(0, \frac{\sigma_{\infty}^{2}(\xi)}{f^{2}(\xi)}\right) .
$$

Observe that for the CLT to hold for strong mixing sequences, we only need that $\alpha(k) \leq C n^{-\theta}$ with $\theta>1+\sqrt{2}$.

Remark 2 Our results also apply for stochastic differential equations and stochastic volatility models discretely observed. Indeed, Genon-Catalot et al. (2000) showed that, under some conditions, these models as well as theirs discrete versions, satisfies geometric $\alpha$ or $\rho$-mixing. Therefore the main hypothesis $H(p, X)$ is then fulfilled for any $p \geq 2$. Regarding GARCH models which are also widely used in financial modeling, we mention that Davis et al. (1999) showed that under conditions on the moment of the innovations and on the Lyapunov exponent associated to the sequence, the squared of the GARCH sequence is geometric $\alpha$-mixing. Hence, our results apply also for GARCH models.

\section{Simulation Studies}

In this section we present some numerical studies which illustrate the conditions under which $\widehat{\operatorname{VaR}}(q)$ converges to $\operatorname{VaR}(q)$. In these simulations, we choose a correlated Gaussian and Pareto sequences. In both cases, we compare the $\operatorname{VaR}(q)$ where $q=0.95$ to the empirical estimate of $\operatorname{VaR}(q)$. For each set of parameters, we run $(M=10000)$ Monte Carlo simulations and compute the mean absolute error $(\operatorname{MAE}(n))$ between $\widehat{\operatorname{VaR}}(q)$ and $\operatorname{VaR}(q)$

$$
\operatorname{MAE}(n)=\frac{1}{M} \sum_{i=1}^{M}\left|\widehat{\operatorname{VaR}}_{(i)}(q)-\operatorname{VaR}(q)\right| .
$$

We also give a confidence interval with level $95 \%$ to the $\operatorname{VaR}(q)$. We consider three different models. First, a correlated Gaussian sequence, then a correlated sequences with Pareto marginal distributions and finally a stochastic volatility model.

\subsection{Case 1: Dependent Gaussian Process}

Let $\left(X_{i}\right)_{0 \leq i \leq n}$ be a Gaussian sequence with zero mean, unit variance and a correlation function given by:

$$
\varrho_{n}(i):=\operatorname{Cov}\left(X_{0}, X_{i}\right)=(1+|i|)^{-\alpha}, i=1, \ldots, n
$$

where $\alpha>0$. The parameter $\alpha$ tunes the strength of dependence. In particular $\alpha=\infty$ corresponds to the i.i.d. sequence, whereas $\alpha=0,\left(\varrho_{n}(i)=1\right)$ gives perfectly correlated sequence.

We study the process:

$$
T_{n}:=\sqrt{n}(\widehat{\operatorname{VaR}}(q)-\operatorname{VaR}(q)) .
$$

We show that for $\alpha>1\left(\Rightarrow \sum_{i=0}^{\infty}\left|\varrho_{n}(i)\right|<\infty\right.$.

$$
T_{n} \longrightarrow{ }_{n \rightarrow \infty}^{d} \mathcal{N}\left(0, \tau_{\infty}^{2}\right)
$$

where $\tau_{\infty}^{2}=\frac{\sigma_{\infty}^{2}(\operatorname{VaR}(q))}{f^{2}(\operatorname{VaR}(q))}$. Here we recall that $\operatorname{VaR}(0.95)=1.6449$.

In Figure 1, we plot the mean absolute error with a $95 \%$ confidence interval as a function of $n$ for different values of $\alpha$ when $q=0.95$. Clearly the $\operatorname{MAE}(n)$ goes to zero when $n$ large, for any $\alpha>0$. The simulations shows that the $\widehat{\operatorname{VaR}}(q)$ is consistent when the correlation parameter $\alpha>0$. When $\alpha>1$, in Figure 2, we plot $\sqrt{n} M A E$ against $n$ to see that it converges to a constant. In Figure 3, we see that the $M A E(n)$ as a function of $\alpha$ for different values of $n$ with $q=0.95$, tends to zero for large values of $n$. In Figure 4, we compare the histogram of $T_{n}$ for $\alpha=3$ and $n=800$ with the density function of Gaussian distribution $\mathcal{N}\left(0, \tau_{\infty}^{2}\right)$. Clearly, for $\alpha>1$ the histogram of $T_{n}$ is close to the normal distribution, confirming our result (5.1).

\subsection{Case 2: Dependent Pareto Process}

We now consider the $\widehat{\operatorname{VaR}}(q)$ for a correlated Pareto sequence $\left(X_{i}\right)_{0 \leq i \leq n}$. Recall that the distribution function of Pareto is defined for $\beta>0$ by:

$$
G_{\beta}(x)= \begin{cases}1-\left(\frac{x_{0}}{x}\right)^{\beta} & x>x_{0} \\ 0 & x \leq x_{0}\end{cases}
$$


To construct a correlated Pareto sequence, we let $X_{i}=G_{\beta}^{-1}\left(\Phi\left(Y_{i}\right)\right)$ where $\Phi$ is the Gaussian distribution $\mathcal{N}(0,1)$ and $\left\{Y_{i}\right\}_{0 \leq i \leq n}$ is a correlated Gaussian sequence defined as in the previous example. As in the first case, we study the process $T_{n}$ to illustrate the central limit theorem (see (5.1)). Here $\operatorname{VaR}(0.95)=2.7144$ when $\beta=3$.

In Figure 5, we plot $\operatorname{MAE}(n)$ with a 95\% confidence interval as a function of $n$ for different values of $\alpha$ when $q=0.95$. Clearly, the MAE goes to zero when $n$ large, for any $\alpha>0$. The simulations shows that the $\widehat{\operatorname{VaR}}(q)$ is consistent when the correlation parameter $\alpha>0$. When $\alpha>1$, in Figure 6, we plot $\sqrt{n} M A E(n)$ against $n$ to see that it converges to a constant. In Figure 7, we see that the $\operatorname{MAE}(n)$ as a function of $\alpha$ for different values of $n$ with $q=0.95$, tends to zero for large values of $n$. In Figure 8, we compare the histogram of $T_{n}$ for $\alpha=3$ and $n=800$, with the density function of Pareto distribution. Here again, when $\alpha>1$, the CLT is satisfied.

\subsection{Case 3: Stochastic Volatility Models}

We assume that $\widehat{V a R}(q)$ of the correlated sequence $\left(X_{i}\right)_{0 \leq i \leq n}$ with stochastic volatility:

$$
X_{i}=\sigma_{i} \cdot \varepsilon_{i}
$$

where $\left(\varepsilon_{i}\right)_{0 \leq i \leq n}$ is an iid Gaussian sequence $\mathcal{N}(0,1)$ and $\left(\sigma_{i}\right)_{0 \leq i \leq n}$ correlated Gaussian or Pareto sequences.

As in the first case, we study the process $T_{n}$ to prove (5.1) where $\operatorname{VaR}(0.95) \approx 1.5949$ for the Gaussian sequence and $\operatorname{VaR}(0.95) \approx 2.4615$ for the Pareto sequence with $\beta=3$. In Figure 9 , we compare the histogram of $T_{n}$ for $\alpha=3$ and $n=800$, with the density function of Gaussian distribution $\mathcal{N}\left(0, \tau_{\infty}^{2}\right)$ using two cases (Gaussian and Pareto for the distribution function of $\left.\sigma_{i}\right)$. Here again, when $\alpha>1$, the CLT is satisfied.

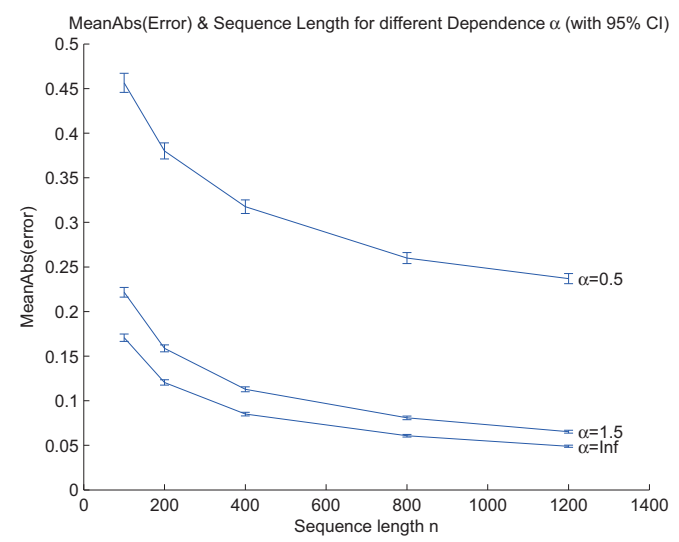

Figure 1. The Mean Absolute Error $(M A E(n))$ with $95 \%$ confidence intervals for correlated Gaussian sequence with correlation function $\varrho_{n}(i)=(1+|i|)^{-\alpha}$ is plotted against the sequence length $n$ for different values of dependence parameter $\alpha$

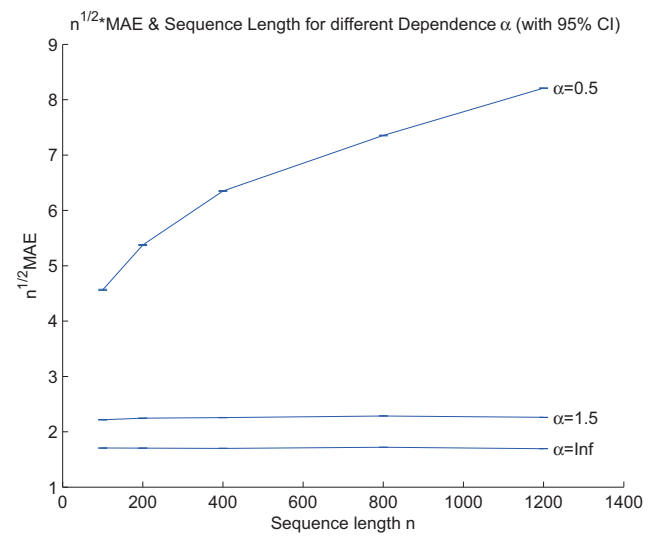

Figure 2. $(\sqrt{n} M A E(n))$ with $95 \%$ confidence intervals for correlated Gaussian sequence with correlation function $\varrho_{n}(i)$ is plotted against the sequence length $n$ for $\alpha \in\{0.5,1.5, \infty\}$. The value $(\sqrt{n} M A E(n))$ tends to a constant for $\alpha>1$ indicating that the optimal convergence rate $O\left(n^{-\frac{1}{2}}\right)$ is achieved 


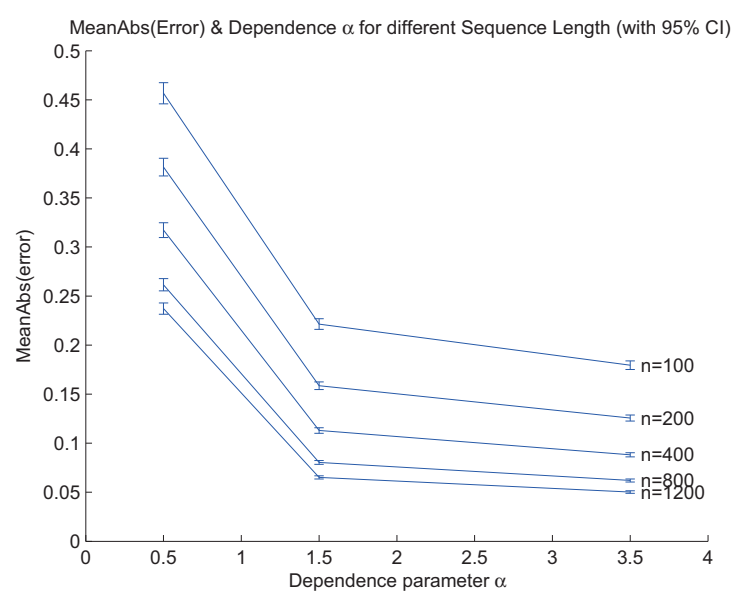

Figure 3. The Mean Absolute Error $(M A E(n))$ with $95 \%$ confidence intervals for correlated Gaussian sequence with correlation function $\varrho_{n}(i)$ is plotted against the dependence parameter $\alpha$ for different values of $n$

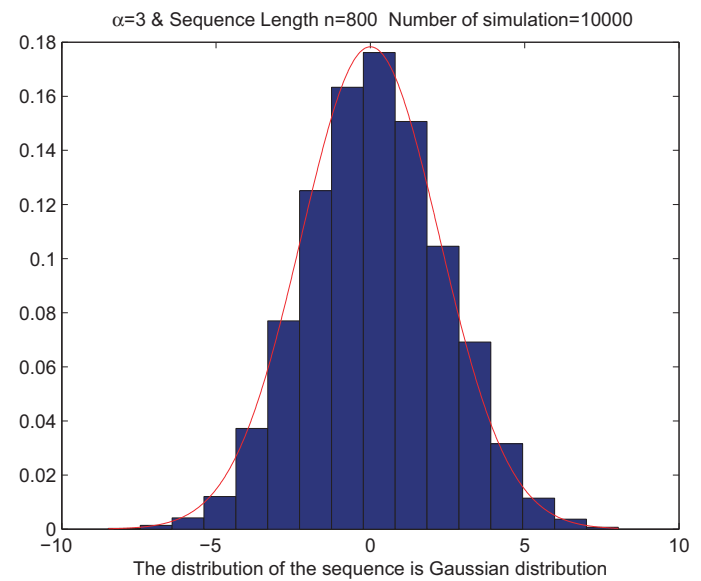

Figue 4. Comparing the histogram of $T_{n}$ for a Gaussian sequence where $\alpha=3$ and $n=800$, with the density function of Gaussian distribution $\mathcal{N}\left(0, \tau_{\infty}^{2}\right)$

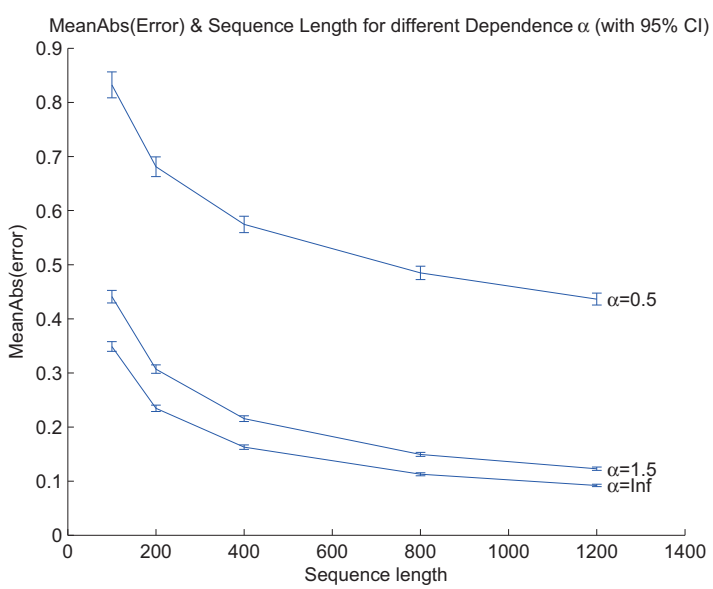

Figure 5. The Mean Absolute Error $(M A E(n))$ with 95\% confidence intervals for correlated Pareto sequence with correlation function $\varrho_{n}(i)$ is plotted against the sequence length $n$ for different values of dependence parameter $\alpha$ 


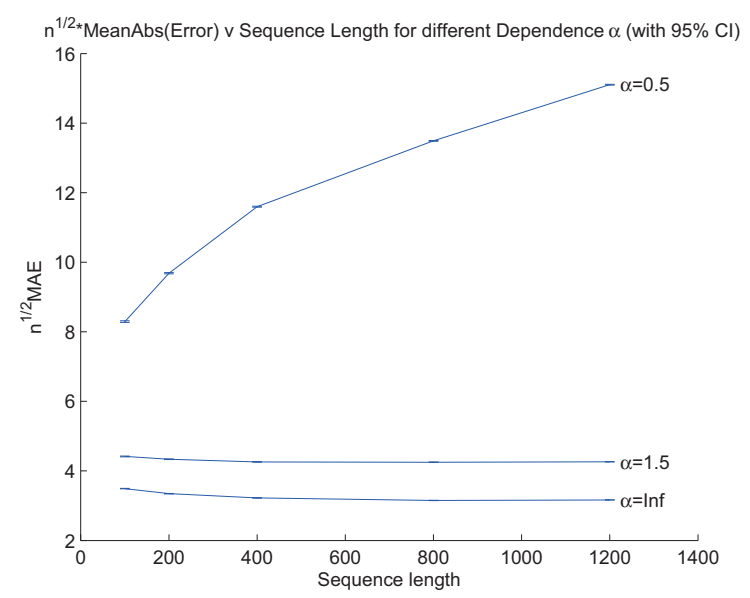

Figure 6. $(\sqrt{n} M A E(n))$ with $95 \%$ confidence intervals for correlated Pareto sequence with correlation function $\varrho_{n}(i)$ is plotted against the sequence length $n$ for $\alpha \in\{0.5,1.5, \infty\}$. The value $(\sqrt{n} M A E(n))$ tends to a constant for $\alpha>1$ indicating that the optimal convergence rate $O\left(n^{-\frac{1}{2}}\right)$ is achieved

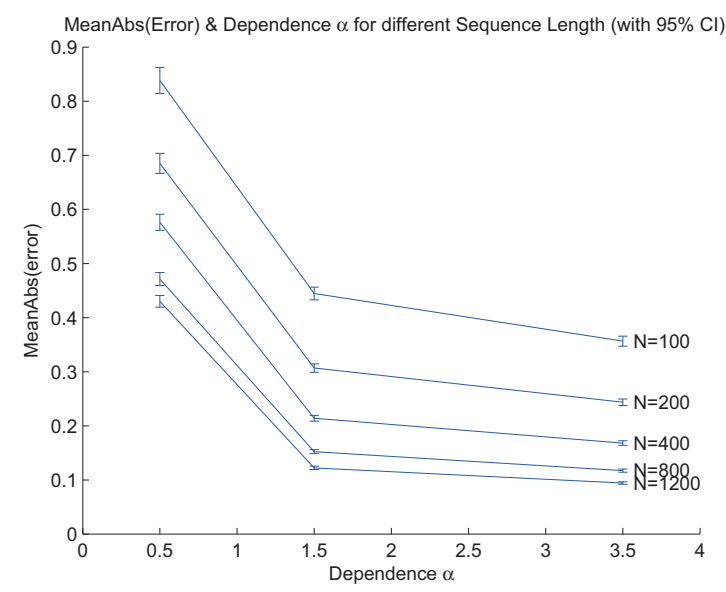

Figure 7. The Mean Absolute Error $(\operatorname{MAE}(n))$ with 95\% confidence intervals for correlated Pareto sequence with correlation function $\varrho_{n}(i)$ is plotted against the dependence parameter $\alpha$ for different values of $n$

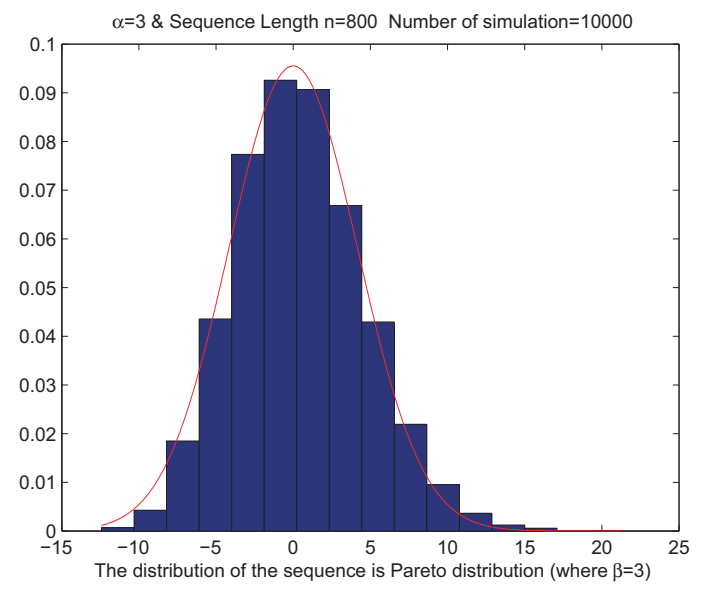

Figure 8. Comparing the histogram of $T_{n}$ for a Pareto sequence where $\alpha=3$ and $n=800$, with the density function of Gaussian distribution $\mathcal{N}\left(0, \tau_{\infty}^{2}\right)$ 

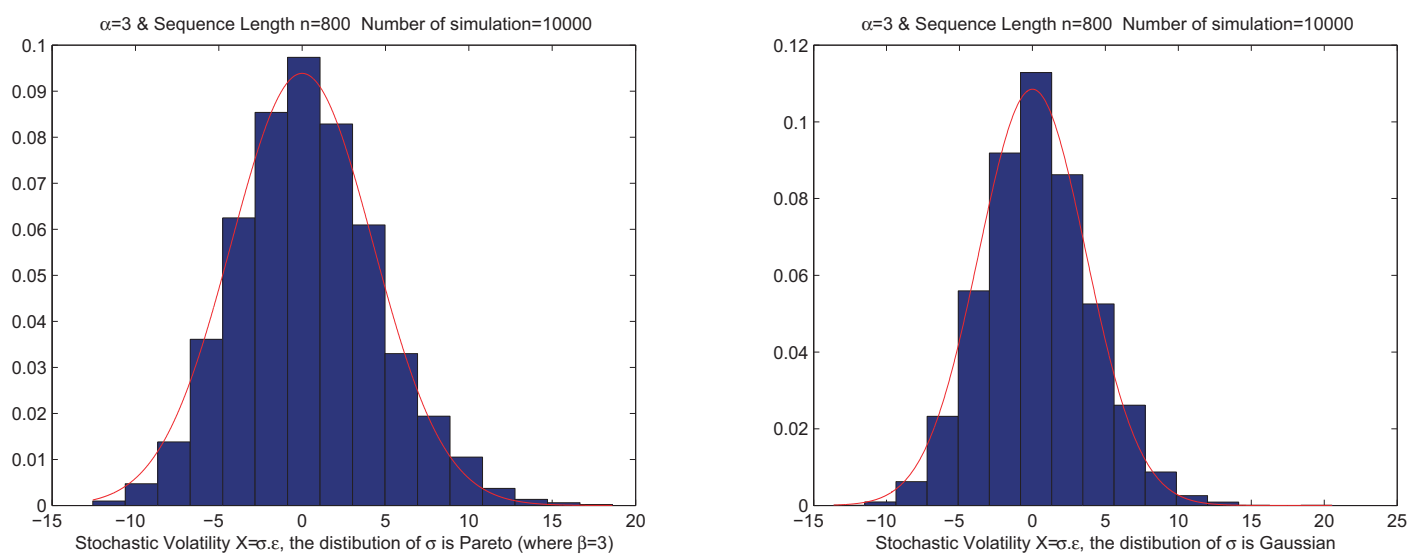

Figure 9. Comparing the histogram of $T_{n}$ for $\alpha=3$ and $n=800$, with the density function of Gaussian distribution $\mathcal{N}\left(0, \tau_{\infty}^{2}\right)$ for two case (Gaussian and Pareto sequence)

\section{Conclusion}

In this work, we considered the nonparametric estimator of the VaR. We proved the consistency of the empirical estimator and a central limit theorem for $\sqrt{n}\left(\xi_{n}-\xi\right)$. Ours results apply as soon as we have a moment inequality for the partial sums. Although the limit is normal like the i.i.d. case, the limiting variance is different and typically larger with dependent observations. One consequence is: the confidence interval for the VaR will be larger. Another question arise about the estimation of this variance. Our results apply for weakly dependent sequences, including mixing sequences, linear process, gaussian sequences and others. It would be interesting to study the estimation of the VaR for long-range dependent sequences.

\section{References}

Ben Hariz, S. (2005). Uniform CLT for empirical process. Stochastic Processes and their Applications, 115(2), 339-358. http://dx.doi.org/10.1016/j.spa.2004.09.006

Ben Hariz, S. (2011). Moment Inequalities for short and long-range dependent sequences. Preprint.

Bennett, G. (1962). Probability Inequalities for the Sum of Independent Random Variables. Journal of the American Statistical Association 57(297), 33-45. http://dx.doi.org/10.2307/2282438

Breuer, P., \& Major, P. (1983). Central limit theorems for nonlinear functionals of Gaussian fields. J. Multivariate Anal., 13(3), 425-441. http://dx.doi.org/10.1016/0047-259X(83)90019-2

Chen, S. X., \& Tang, C. Y. (2005). Nonparametric Inference of Value at Risk for dependent Financial Returns. Journal of Financial Econometrics, 3, 227-255.

Cont, R. (2001). Empirical properties of asset returns: Stylized facts and statistical issues. Quant. Finance, 1, 223-236. http://dx.doi.org/10.1080/713665670

Cramér, H. (1946). Mathematical Methods of Statistics. Princeton, New Jersey: Princeton University Press.

Csörgo, S., \& Mielniczuk, J. (1996). The empirical process of a short-range dependent stationary sequence under Gaussian subordination. Probab. Theory Related Fields, 104(1), 15-25. http://dx.doi.org/10.1007/BF01303800

Davis, R. A., Mikosch, T., \& Basrak, B. (1999). Sample ACF of multivariate stochastic recurrence equations with applications to GARCH. Preprint.

Dowd, K. (2001). Estimating VaR with order statistics. Journal of Derivatives, 23-30. http://dx.doi.org/10.3905/jod.2001.319154

Hoeffding, W. (1963). Probability Inequalities for Sums of Bounded Random Variables. Journal of the American Statistical Association, 58(301), pp. 13-30.

Hu, S. H. (2003). Some new results for the strong law of large numbers. Acta Mathematica Sinica, 46, 1123-1134.

Lahiri, S. N., \& Sun, S. (2009) A Berry-Esseen theorem for sample quantiles under weak dependence. Ann. Appl. 
Probab., 19(1), 108-126. http://dx.doi.org/10.1214/08-AAP533

Marinelli, C., d'Addona, S., \& Rachev, S. T. (2007). A comparison of some univariate models for value-at-risk and expected shortfall. Int. J. Theor. Appl. Finance, 10(6), 1043-1075. http://dx.doi.org/10.1142/S0219024907004548

Peligrad, M. (1985). An invariance principle for $\varphi$-mixing sequences. Ann. Probab. 13(4), 1304-1313. http://dx.doi.org/10.1214/aop/1176992814

Rio, E. (1997). Théorèmes limites pour des variables aléatoires faiblement dépendentes. Preprint, Universit de Paris Sud, no. 97/81.

Sen, P. K. (1972). On the Bahadur representation of sample quantiles for sequences of $\phi$-mixing random variables. J. Multivariate Anal., 2, 77-95.

Shao, Q., \& Yu, H. (1996). Weak convergence for weighted empirical processes of dependent sequences. Ann. Probab., 24(4), 2098-2127. http://dx.doi.org/10.1214/aop/1041903220

Utev, S., \& Peligrad, M. (2003). Maximal inequalities and an invariance principle for a class of weakly dependent random variables. J. Theoret. Probab., 16(1), 101-115.

Valentine Genon-Catalot, Thierry Jeantheau, \& Catherine Larédo. (2000). Stochastic Volatility Models as Hidden Markov Models and Statistical Applications. 1051-1079. http://dx.doi.org/10.2307/3318471

Van der Vaart, Aad W., \& Wellner, Jon A. (1996). Weak convergence and empirical processes. With applications to statistics. Springer Series in Statistics. New York: Springer-Verlag. ISBN: 0-387-94640-3. 